\title{
Towards quantum simulation of Sachdev-Ye-Kitaev model
}

\author{
Ye Cao, ${ }^{1}$ Yi-Neng Zhou, ${ }^{2}$ Ting-Ting Shi, ${ }^{2}$ and Wei Zhang ${ }^{2,3, *}$ \\ ${ }^{1}$ School of Physics, Beijing Institute of Technology, Beijing 100081, China \\ ${ }^{2}$ Department of Physics, Renmin University of China, Beijing 100872, China \\ ${ }^{3}$ Beijing Key Laboratory of Opto-electronic Functional Materials and Micro-nano Devices, \\ Renmin University of China, Beijing 100872, China
}

(Dated: March 4, 2020)

\begin{abstract}
We study a simplified version of the Sachdev-Ye-Kitaev (SYK) model with real interactions by exact diagonalization. Instead of satisfying a continuous Gaussian distribution, the interaction strengths are assumed to be chosen from discrete values with a finite separation. A quantum phase transition from a chaotic state to an integrable state is observed by increasing the discrete separation. Below the critical value, the discrete model can well reproduce various physical quantities of the original SYK model, including the volume law of the ground-state entanglement, level distribution, thermodynamic entropy, and out-of-time-order correlation (OTOC) functions. For systems of size up to $N=20$, we find that the transition point increases with system size, indicating that a relatively weak randomness of interaction can stabilize the chaotic phase. Our findings significantly relax the stringent conditions for the realization of SYK model, and can reduce the complexity of various experimental proposals down to realistic ranges.
\end{abstract}

PACS numbers: 05.30.Fk, 03.75.Hh, 03.75.Ss, 67.85.-d

\section{INTRODUCTION}

Since 1970s, models of infinite-range random interacting particles have been introduced and gained widespread attention in condensed matter physics [1-7]. The ground states of these models are believed to break long-range order and be non-Fermi liquid or spin-glass. Recently, Kitaev has proposed an exactly solvable model [8] equivalent to Sachdev and Ye's proposal [2] in the sense of identical saddle-point equations in the large- $N$ limit. This so-called Sachdev-Ye-Kitaev (SYK) model, as defined in Eq. (1), springs up in a wide range of areas since it is believed to be a holographic dual of the $\mathrm{AdS}_{2}$ horizons of charged black holes and intimately related to various important topics, including quantum chaos, eigenstate thermal hypothesis (ETH), and information theory, to name a few [9-17]. Inspired by the prototype, some variant models were proposed to explore (topological) phase transitions [18-23], the stability of nonFermi liquid phase [24-29], the properties in high dimensions $[19,25,26]$, as well as level statistics and many-body localization [30, 31].

Alongside the exciting theoretical progresses, the experimental realization of the SYK model is still hindered mainly by the complicated form of interactions. Recently, a few proposals have been put forward in different physical platforms. In condensed matter systems, there are suggestions using Majorana zero modes residing on the surface of a topological insulator [32] or hosted by topological superconducting wires coupled with a quantum dot [33]. Besides, a device without the requirement of superconductivity is proposed in an irregular shaped

*Electronic address: wzhangl@ruc.edu.cn graphene flake [34], where the transport characteristics are further examined theoretically [35]. Another possible route towards this goal is to employ the high controllability of ultracold quantum gases. Danshita et al. have presented a variant of SYK model [36], which can be realized in principle by coupling two confined fermionic atoms with molecular states via photo-association lasers. In all these proposals, much effort has been devoted to fulfill the three conditions of the SYK model: (i) the inter-site tunneling terms are absent, (ii) all particles are coupled via an infinitely long-range four-fermion interaction $J_{i j k l}$, and (iii) the interaction strengths of $J_{i j k l}$ are distributed in a Gaussian form. While the tunneling between sites can be turned off rather easily in some proposals, the latter two points require very delicate and complex setups which are too difficult to be implemented in experiments for systems of only a few particles. An important question then naturally arises: Whether and to what extent the stringent requirement of a perfectly random all-to-all interaction can be relaxed to simulate the SYK model?

In this work, we consider a significantly simplified variant of SYK model, where the random interaction strengths can only take real and discrete values separated by a give distance, i.e., $0, \pm d, \pm 2 d, \ldots$ The probability for the interaction to take a specific value renders a Gaussian distribution, hence for large enough separation $d$, the interaction is very likely to be zero and the majority of particles become mutually non-interacting. This model significantly relaxes the stringent conditions (ii) and (iii) aforementioned, and paves the route towards the experimental simulation of SYK model by reducing the complexity in a large extent. Using exact diagonalization, we investigate the level distribution of the spectrum and find a quantum phase transition from an SYK-like phase to a Fermi liquid phase by increasing the separation $d$ of inter- 
action strength. This transition leaves clear signatures in various physical properties, including the entanglement entropy of the ground state, the thermodynamic entropy at finite temperature, and the out-of-time-ordered correlation (OTOC) function. For system size up to $N=20$, we find that the transition point increases with $N$, which means for larger systems the conditions (ii) and (iii) can be further relaxed and be more easily implemented.

\section{MODEL HAMILTONIAN}

The SYK model in $0+1$ space-time dimensions is governed by a model of spinless fermions on a onedimensional lattice:

$$
\mathcal{H}_{\mathrm{SYK}}=\frac{1}{(2 N)^{\frac{3}{2}}} \sum_{i, j, k, l}^{N} J_{i j, k l} c_{i}^{\dagger} c_{j}^{\dagger} c_{k} c_{l}-\mu \sum_{i} c_{i}^{\dagger} c_{i},
$$

where $c_{i}^{\dagger}$ are fermion creation operators and $J_{i j, k l}$ are complex random couplings satisfying a Gaussian distribution with zero mean. The interaction has the following properties

$$
\begin{aligned}
& J_{i j, k l}=-J_{j i, k l}, \quad J_{i j, k l}=-J_{i j, l k}, \\
& J_{i j, k l}=-J_{k l, i j}^{*}, \quad \overline{\left|J_{i j, k l}\right|^{2}}=J^{2} .
\end{aligned}
$$

The original SYK model Eq. (1) constituted of complex interactions contains both amplitude and phase randomness, hence is in principle hard to realize experimentally. To overcome this difficulty, we consider a variant model by setting the couplings to be real and take values separated by a specific step, i.e.,

$$
\tilde{J}_{i j, k l}=\operatorname{round}\left(J_{i j, k l} / d\right) \times d,
$$

where $J_{i j, k l}$ are real Gaussian random couplings distributed as

$$
\begin{aligned}
& J_{i j, k l}=-J_{j i, k l}, \quad J_{i j, k l}=-J_{i j, l k}, \\
& J_{i j, k l}=-J_{k l, i j}, \quad \overline{\left|J_{i j, k l}\right|^{2}}=J^{2},
\end{aligned}
$$

and the round function returns the value of its argument rounded to the nearest integer. The Hamiltonian finally becomes

$$
\mathcal{H}^{\mathrm{dis}}=\frac{1}{(2 N)^{\frac{3}{2}}} \sum_{i, j, k, l}^{N} \tilde{J}_{i j, k l} c_{i}^{\dagger} c_{j}^{\dagger} c_{k} c_{l}-\mu \sum_{i} c_{i}^{\dagger} c_{i} .
$$

From now on, we denote the original SYK Hamiltonian Eq. (1) as complex-SYK model, and the variation of original SYK model where the couplings are replaced with real Gaussian random numbers is referred to as real-SYK model [38]. Meanwhile, the Hamiltonian in Eq. (5) is referred as discrete-real-SYK model, which recovers the continuous-real-SYK model as the discrete distance approaches to zero. In the opposite limit of large discrete distance $d$, most of the random numbers are rounded
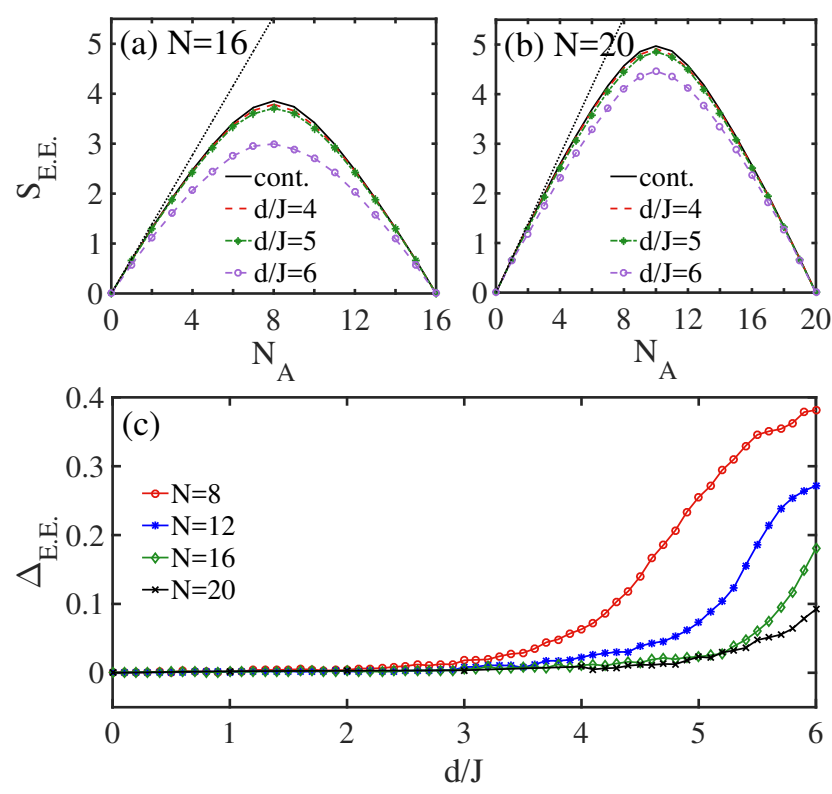

Figure 1: (Color online) Entanglement entropy $\left(S_{\text {E.E. }}\right)$ of a subsystem of size $N_{A}$ in a lattice of size (a) $N=12$ and (b) $N=16$. The relative difference $\Delta_{\text {E.E. }}$. between the discretereal-SYK model and the continuous-SYK model (both real and complex) for different choices of discrete distance $d / J$ are shown in (c).

to zero, making the Hamiltonian returns into a noninteracting model. Next, we use exact diagonalization to study the three models and compare results for various properties. As the outcome of the complex-SYK model and the continuous-real-SYK model are very close for all system sizes we have considered, in the following discussion we do not distinguish the two cases and refer to them as continuous (cont.) models.

\section{GROUND STATE AND LEVEL SPECTRUM}

A remarkable property of the complex-SYK model is the non-Fermi liquid ground state with volume law of bipartite entanglement, which is the first quantity we explore here. The problem we are interested in is to what extent does the basic property of the complex-SYK model remains. Firstly, we focus on the zero-temperature properties, so only a sector of Hilbert space with fixed particle number should be taken into consideration. For numerical convenience, we choose the half-filling sector and carry out calculation for system size $N$ a multiple of 4 .

In Fig. 1, we present the ground-state entanglement entropy for the discrete-real-SYK model by varying the discrete separation $d$. The entanglement entropy $\left(S_{\mathrm{E} . \mathrm{E} .}\right)$ is obtained by calculating the von Neumann entropy of 
the reduced density matrix of subsystem denoted as $A$,

$$
\begin{aligned}
S_{\mathrm{E} . \mathrm{E}} & =-\operatorname{Tr}\left(\rho_{\mathrm{A}} \ln \rho_{\mathrm{A}}\right), \\
\rho_{\mathrm{A}} & =\operatorname{Tr}_{\overline{\mathrm{A}}}(|G\rangle\langle G|),
\end{aligned}
$$

where $\{A, \bar{A}\}$ partitions the spatial freedoms of the lattice. Specifically, we choose $N_{\mathrm{A}}$ consecutive sites on the left as the subsystem, $\rho_{\mathrm{A}}$ is the corresponding reduced density matrix, and $|G\rangle$ the ground state of Hamiltonian Eq. (5). As shown by black solid lines in Figs. 1(a) and 1(b), the entanglement entropy density of continuousSYK model (both complex and real) comes between $\ln 2$ (dotted line) and the analytic result 0.464848 in zerotemperature limit [37].

We then compare the result with the outcome of discrete models. When the separation $d / J$ between the possible strengths of random interactions is small, the entanglement entropy obtained from the discrete model gets reduced from the continuous model, with a very slight deviation for all lattice sizes up to the largest value we have considered $N=20$, as shown in Figs. 1(a) and 1(b). With increasing $d / J$, the discrepancy becomes more evident and the entanglement entropy is further reduced. This tendency is naturally expected since the system approaches to a non-interacting model in the limiting case of large $d / J$. However, an interesting and important finding is that the deviation between the discrete and continuous models is not linearly dependent on $d / J$. To further quantify this observation, we calculate the relative difference between curves of the discrete and continuous models by summing over lattice points except the end points where the entanglement entropy is trivially zero

$$
\Delta_{\text {E.E. }} \equiv \frac{1}{N_{A}} \sum_{N_{A}} \frac{\left|S_{\text {E.E. }}^{\text {(disc.) }}-S_{\mathrm{E} . \mathrm{E}}^{\text {(cont.) }}\right|}{S_{\mathrm{E} . \mathrm{E}}^{\text {(cont.) }}} .
$$

The superscript disc. and cond. stand for results from the discrete and continuous models, respectively. As shown in Fig. 1(c), in the regime where the distance $d / J$ is small, the relative difference nearly remains zero, indicating that the continuous model can be well approximated by the discrete models. When the separation exceeds a critical value, the discrepancy is rapldly enhanced and presents a transition-like behavior. The critical value also increases with the system size, suggesting that for larger systems, a fewer number of random interaction strengths are required to mimic the SYK model. Since the system is reduced to a non-interacting model with $d / J \rightarrow \infty$, one would expect that the critical threshold should converge to an asymptotic value in the thermodynamic limit, which, however, can not be extracted from our numerical results since the calculation is very costly and can only be restricted to systems with $N \leq 20$.

The results shown in Fig. 1 are averaged over a few tens sampling systems. To check convergence, we exhibit the averaged results for different sampling numbers, as illustrated in Fig. 2. Comparing to models with only short-range interactions, the ground state of SYK model
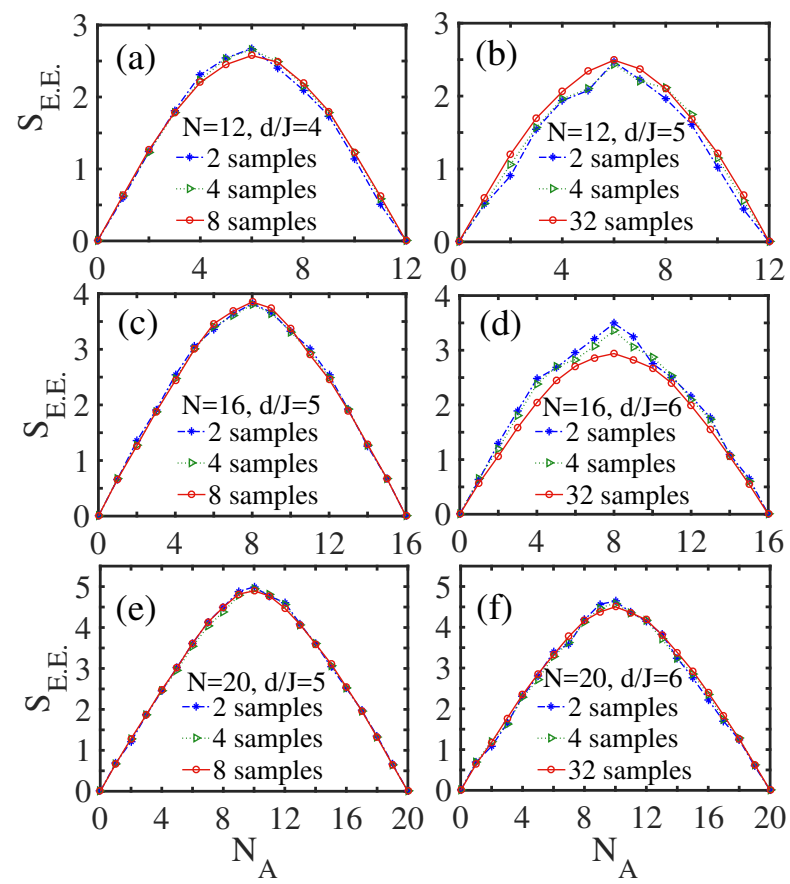

Figure 2: (Color online) Convergence of the entanglement entropy before (left column) and after (right column) the transition. For $d / J$ larger than the critical value, more samples are required to approach the averaged value. This observation suggests that the system is no longer fully thermalized, as it does in a chaotic phase with small $d / J$.

is believed to be thermalized, which means that the results for any random samplings are almost the same in a sufficiently large system. Indeed, for the continuous-realSYK model with 12 or more sites, the numerical result of entanglement entropy of one specific sampling is already very close to the converged average. For discrete models, as the interaction can only choose from a finite set of values, with a large probability to be zero in particular, the variance of a given sampling is more evident. When the separation $d / J$ is less than the critical value, the convergence can still be quickly obtained for a few samplings, as depicted in Figs. 2(a), 2(c), and 2(e). However, in the regime above the threshold as in Figs. 2(b), 2(d) and $2(\mathrm{f})$, the convergence becomes fairly slow and one has to average over more than a few tens of samples. This result implies the existence of a threshold as a function of system size in the perspective of ETH, in consistence with the trend extracted from entanglement entropy shown in Fig. 1(c).

To characterize the nature of this phase transition, we then analyze the energy spectrum of the system. For the original SYK model, the ground state is the maximally chaotic non-Fermi liquid phase, with an energy spectrum obeying one of the three chaotic ensembles depending on the number of particles. [13,14] The three ensembles correspond to quantum Hamiltonians of random matrices whose entries are real (GOE), complex (GUE), or quaternionic (GSE) variables, For the specific filling 

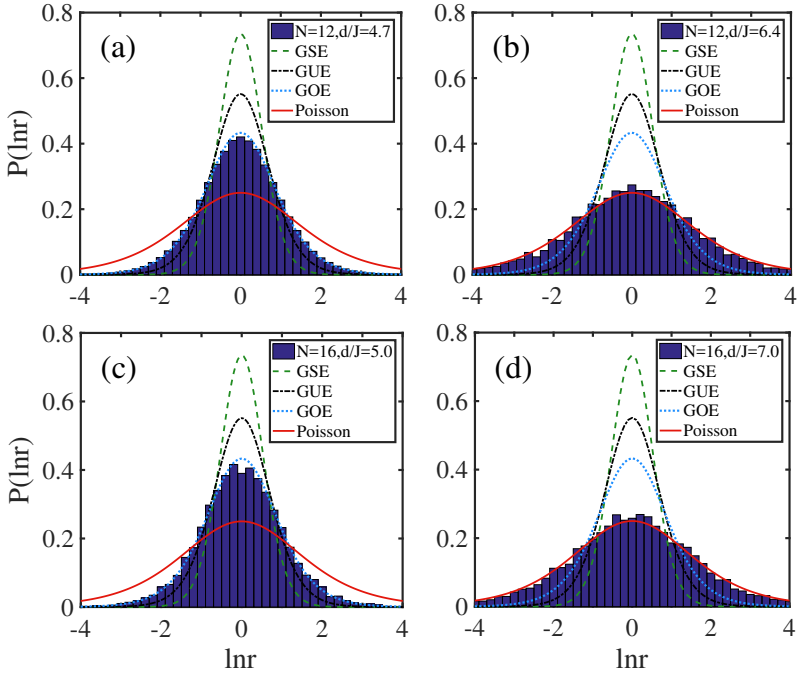

Figure 3: (Color online) Level statistics (blue histogram) of the discrete model before (left column) and after (right column) the transition. The system clearly evolves from a chaotic state with Wiger-Dyson distribution (GOE) at small $d / J$ to an integrable state with Poisson distribution at large $d / J$.

factor considered in the present case, the many-body energy spectrum of the original SYK model falls into the GOE class. By ordering the energy levels from bottom to top, we can define the ratio between successive spacings [39, 40]

$$
r_{n}=\frac{E_{n+1}-E_{n}}{E_{n+2}-E_{n+1}},
$$

which is predicted to be distributed according to the following function

$$
P(r)=\frac{1}{Z} \frac{\left(r+r^{2}\right)^{\beta}}{\left(1+r+r^{2}\right)^{1+3 \beta / 2}}
$$

with Dyson index $\beta=1$ and normalization constant $Z=$ $8 / 27$.

In Fig. 3, we show the histogram of $r_{n}$ for the discretereal-SYK model at typical separations $d / J$ before and after the transition for systems of size $N=12$ and 16 . For the cases of small $d / J$ as in Figs. 3(a) and 3(c), the distribution $P(r)$ obeys the prediction for GOE, indicating that the system is in a quantum chaotic phase. After crossing the transition point, however, the level distribution approaches to a Poisson statistics $P(r)=e^{-r}$, which corresponds to an integrable quantum system. A similar chaotic-integrable transition is investigated in another variant of SYK model with an additional one-body infinite-range random interaction. [15] Here, we show that such a transition can be driven by simply reducing the randomness of the two-body interaction, without introducing any other mechanisms.

Another characteristic quantity of the level statistics is the average ratio between the smallest and the largest

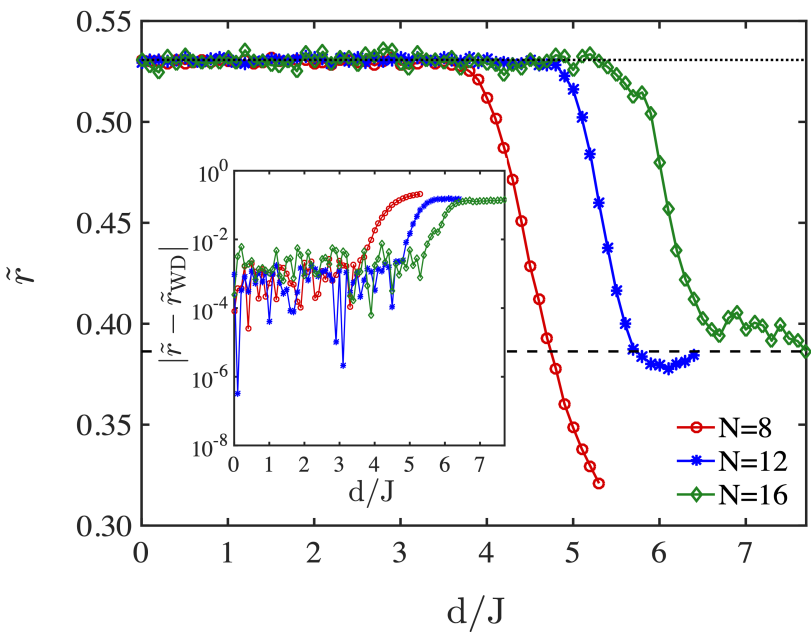

Figure 4: (Color online) Average ratio of adjacent energy gaps as a function of separation $d / J$. Below the critical threshold, the results for all system sizes recover the value 0.5307 for a chaotic state. By crossing the transition point, the average ratio starts to drop and saturates to the limiting value of 0.3863 of Poisson statistics. Inset depicts the same plots in the logarithmic scale, showing that the deviation from the chaotic state is indeed a sharp transition rather than an exponential enhancement.

adjacent energy gaps [39]

$$
\tilde{r}_{n}=\frac{\min \left[\delta_{n}^{E}, \delta_{n-1}^{E}\right]}{\max \left[\delta_{n}^{E}, \delta_{n-1}^{E}\right]} .
$$

Here, $\left\{E_{n}\right\}$ is the ordered list of energy levels and $\delta_{n}^{E}=$ $E_{n}-E_{n-1}$ is the separation between two adjacent eigenstates. From Fig. 4, we find that when $d / J$ is smaller than the critical threshold for a give system size, the average ratio recovers the result $\langle\tilde{r}\rangle \equiv r_{\mathrm{WD}} \approx 0.5307$ (dotted line) of a Wigner-Dyson distribution for GOE. If the separation $d / J$ exceeds the critical value, the average ratio $\tilde{r}$ deviates significantly from that of a thermal state, and approaches to the limiting value of $2 \ln 2-1 \approx 0.3863$ (dashed line) for a Poisson distribution in large enough systems. For the smallest system $N=8$, the result of $\tilde{r}$ goes below the Poisson limit and does not show any clear saturation for the largest value of $d / J$ we have tried. In fact, for such a small system with $d / J \gtrsim 5$, most of the interaction terms $(\gtrsim 98.7 \%)$ are tuned off and the system presents many nearly degenerate energy levels, which compromise the definition and determination of $\tilde{r}$. We also plot the difference of $\tilde{r}$ from the Wigner-Dyson result $\tilde{r}_{\mathrm{WD}}$ in a logarithmic scale in the inset of Fig. 4, from which one can conclude that the deviation from the chaotic state is indeed a quantum phase transition. The transition points for specific lattice sizes agree well with the result of entanglement entropy as in Fig. 1(c). 


\section{FINITE TEMPERATURE PROPERTIES}

Next, we probe the tolerance to discretization at finite temperature. For a physical quantity $f$, we define the relative difference from the continuous result as

$$
\Delta_{f} \equiv \frac{\int_{a}^{b} d x\left[f^{\text {(disc. })}(x)-f^{(\text {cont. })}(x)\right]}{\int_{a}^{b} d x f^{\text {(cont. })}(x)}
$$

where $a(\mathrm{~b})$ is the lower (upper) bound of the parameter range, and $f^{\text {(disc.) }}\left(f^{\text {(cont. })}\right)$ is a function to be evaluated for the discrete (continuous) SYK model.

We first examine the thermodynamic entropy, which can be expressed as

$$
\frac{S}{N}=\frac{\langle E\rangle / T+\ln \mathcal{Z}}{N}
$$

Here, the partition function $\mathcal{Z}$ reads $\mathcal{Z}=\sum_{n} e^{-E_{n} / T}$ with $E_{n}$ the eigenvalues for the canonical ensemble, and $\langle E\rangle=(1 / \mathcal{Z}) \sum_{n} E_{n} e^{-E_{n} / T}$ is the average energy. Again, we find that the discrete-real-SYK model can mimic the original model provided that the separation $d / J$ is smaller than the critical value. However, the transition is not as sharp as in Figs. 1 and 4, and does not show a clear enhancement with system size. The relative difference becomes significant when $d / J \gtrsim 4$ for both $N=12$ and 16, as shown in Figs. 5(a) and 5(c). Another observation is that the relative difference first becomes negative and then increases with $d$ on the rise. Therefore, in the perspective of relative difference, there is a specific value for $d / J \approx 3.5$ which can mostly recover the original SYK model.

Finally, we analyze the exponential decay of the outof-time-ordered correlation (OTOC) which is closely related to the quantum butterfly effect. The decay rate $\lambda_{L}$ is known as Lyapunov exponent in classical chaos theory and depicts the strength of chaos. The upper bound of $\lambda_{L}$ is determined in the strong-coupling limit as $2 \pi / \beta$ with $\beta$ the inverse temperature, and is argued to be reached in the SYK model for strongly interacting Majorana fermions in the thermodynamic limit, as well as in the theory of Einstein gravity on the $\mathrm{AdS}_{2}$ horizons of blackholes. [11, 37] However, in finite-size calculations, the theoretical results can not be reproduced in the strong coupling limit and $\lambda_{L}$ is not sensitive to $\beta J$ at finite temperatures [37]. Here, we define a normalized OTOC as

$$
\mathcal{O}(t)=-\frac{\operatorname{Re}\langle A(t) B(0) A(t) B(0)\rangle_{\beta}}{\langle A(0) A(0)\rangle_{\beta}\langle B(0) B(0)\rangle_{\beta}},
$$

where $A$ and $B$ are Majorana operators

$$
\begin{aligned}
& A=c_{1}+c_{1}^{\dagger}, \\
& B=c_{2}+c_{2}^{\dagger} .
\end{aligned}
$$

Since the Lyapunov exponent is not sensitive to the coupling strength, we choose a moderate value $\beta J=1$ and
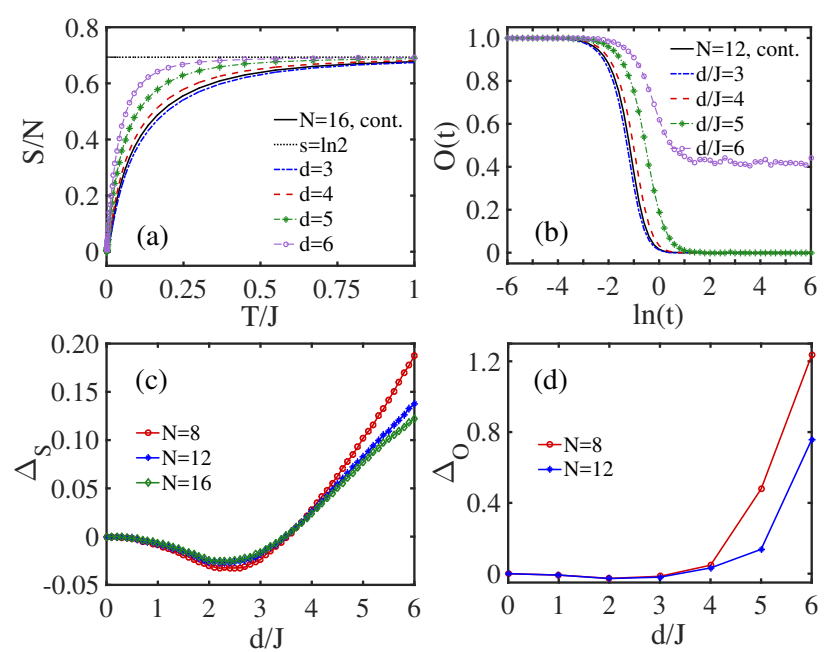

Figure 5: (Color online) (a) Thermal entropy density as a function of temperature with different discretization of couplings for $N=16$, and (c) the relative differences from continuous model. Results for all configurations approach the high temperature limit $S / N=\ln 2$, however, analytical result for the zero-temperature limit can not be obtained for finite size calculation. (b) OTOC as a function of time on logarithmic scales at $T / J=1$ with different discretization of couplings for $N=12$, and (d) its relative difference from the continuous model.

investigate the influence of discretization as shown in Fig. 5.

In line with expectations, the scrambling of OTOC almost keeps unchanged with small $d$. However, when $d / J$ goes beyond a similar threshold, the deviation becomes prominent as shown in Fig. 5(b). Importantly, we find that $\mathcal{O}(t)$ evolves to a finite value rather than zero in the long-time limit. This observation can be understood by noticing that as the couplings become more discretized, most of them are rounded to zero, in which case the Hamiltonian commutes with the Majorana operators. If the commutative case takes a non-zero measure among the samplings, information can be preserved during forward and backward evolution and $\mathcal{O}(t)$ does not converge to zero. In Fig. $5(\mathrm{~d})$, we also show the relative difference between the discrete and continuous models as defined in Eq. (12). A transition-like behavior is also observed when $d / J$ exceeds a critical value, which seems to increase with system size.

\section{SUMMARY AND DISCUSSION}

In this paper, we numerically study a simplified variant of the Sachdev-Ye-Kitaev (SYK) model where the all-toall random two-body interaction can only take real values which are discretely separated by a finite distance $d$. Using exact diagonalization, we numerically solve this discrete model and compare results with the original SYK model for systems of size up to $N=20$ at half filling. 
By increasing the separation $d$, or equivalently reduces the randomness of the interaction, we observe a quantum phase transition from chaotic to integrable states, which can be signatures in various physical quantities including entanglement entropy, level spectrum, thermodynamic entropy, and out-of-time-ordered correlation. The transition point increases with system size, indicating that for larger systems, a chaotic state can be stabilized with a relatively weak randomness. These results extend our knowledge about the SYK model, and about the more general topic of quantum chaotic system.

More importantly, our study paves the way towards the experimental simulation of the SYK model by significantly relaxing the requirement of the interaction. First, we show that the interaction does not to be of infinite range. In fact, most of the inter-particle combinations can be assumed to be non-interacting while the system still reserves the behavior of the SYK model. For instance, in a system of size $N=8$, a discrete model with separation of $d / J=3$ can mimic the original SYK model in all aspects, where only $9.5 \%$ of the total $8 !=1168$ possible interaction terms are non-zero. As the critical transition point increases with the lattice size, this condition is further relaxed for larger systems. Another advantage of our model is that the interaction strength can only take a few possible choices, rather than a completely random distribution. In previous experimental proposals, much effort has been devoted to achieve the desired randomness by either fabricating an irregular sample [32,34] or applying multiple laser beams [36], which are all experimentally challenging. In a discrete model with $d / J=3$, one only needs to take into account at most four possible choices of $\pm d / J$ and $2 \pm d / J$, with the probabilities of $9.0 \%$ and $0.45 \%$, respectively. This can significantly reduce the complexity of experiments.

\section{Acknowledgments}

We thank M. Tezuka and N.-H. Tong for helpful discussion. This work is supported by the National Natural Science Foundation of China (Grants No. 11434011, 11522436, 11774425, 11704029), the National Key R\&D Program of China (Grants No. 2018YFA0306501), the Beijing Natural Science Foundation (Grant No. Z180013), and the Research Funds of Renmin University of China (Grants No. 16XNLQ03 and 18XNLQ15).
[1] D. Sherrington and S. Kirkpatrick, Phys. Rev. Lett. 35, 1792 (1975).

[2] S. Sachdev and J. Ye, Phys. Rev. Lett. 70, 3339 (1993).

[3] O. Parcollet and A. Georges, Phys. Rev. B 59, 5341 (1999).

[4] A. Georges, O. Parcollet, and S. Sachdev, Phys. Rev. Lett. 85, 840 (2000).

[5] A. Georges, O. Parcollet, and S. Sachdev, Phys. Rev. B 63, 134406 (2001).

[6] L. Arrachea and M. J. Rozenberg, Phys. Rev. B 65, 224430 (2002).

[7] A. Camjayi and M. J. Rozenberg, Phys. Rev. Lett. 90, 217202 (2003).

[8] A. Y. Kitaev, A simple model of quantum holography, in KITP strings seminar and Entanglement 2015 program, UC Santa Barbara, Santa Barbara USA, 12 February, 7 April and 27 May 2015, http://online.kitp.ucsb.edu/ online/entangled15/.

[9] S. Sachdev, Phys. Rev. X 5, 041025 (2015).

[10] J. Maldacena, S. H. Shenker, and D. Stanford, J. High Energy Phys. 8, 106 (2016).

[11] P. Hosur, X.-L. Qi, D. A. Roberts, and B. Yoshida, J. High Energy Phys. 2, 4 (2016).

[12] J. Polchinski and V. Rosenhaus, J. High Energy Phys. 4, 1 (2016).

[13] A. M. García-García and J. J. M. Verbaarschot, Phys. Rev. D 94, 126010 (2016).

[14] Y.-Z. You, A.W. W. Ludwig, and C. Xu, Phys. Rev. B 95, 115150 (2017).

[15] A. M. García-garcía, B. Loureiro, A. Romero-bermúdez, and M. Tezuka, Phys. Rev. Lett. 120, 241603 (2018).

[16] T. Scaffidi and E. Altman, Phys. Rev. B 100, 155128 (2019).
[17] A. M. García-garcía, T. Nosaka, D. Rosa, and J. J. M. Verbaarschot, Phys. Rev. D 100, 26002 (2019).

[18] P. Zhang and H. Zhai, Phys. Rev. B 97, 201112 (2018).

[19] S. Banerjee and E. Altman, Phys. Rev. B 95, 134302 (2017).

[20] Z. Bi, C.-M. Jian, Y.-Z. You, K. A. Pawlak, and C. Xu, Phys. Rev. B 95, 205105 (2017).

[21] O. Can and M. Franz, Phys. Rev. B 100, 45124 (2019).

[22] A. Altland, D. Bagrets, and A. Kamenev, Phys. Rev. Lett. 123, 106601 (2019).

[23] H. Guo, Y. Gu, and S. Sachdev, Phys. Rev. B 100, 045140 (2019).

[24] A. M. García-García and J. J. M. Verbaarschot, Phys. Rev. D 96, 066012 (2017).

[25] S.-K. Jian and H. Yao, Phys. Rev. Lett. 119, 206602 (2017).

[26] C.-M. Jian, Z. Bi, and C. Xu, Phys. Rev. B 96, 115122 (2017).

[27] X.-Y. Song, C.-M. Jian, and L. Balents, Phys. Rev. Lett. 119, 216601 (2017).

[28] X. Chen, R. Fan, Y. Chen, H. Zhai, and P. Zhang, Phys. Rev. Lett. 119, 207603 (2017).

[29] T. Krajewski, M. Laudonio, R. Pascalie, and A. Tanasa, Phys. Rev. D 99, 126014 (2019).

[30] E. Iyoda, H. Katsura, and T. Sagawa, Phys. Rev. D 98, 086020 (2018).

[31] A. M. García-García and M. Tezuka, Phys. Rev. B 99, 054202 (2019).

[32] D. I. Pikulin and M. Franz, Phys. Rev. X 7, 031006 (2017).

[33] A. Chew, A. Essin, and J. Alicea, Phys. Rev. B 96, 121119 (2017).

[34] A. Chen, R. Ilan, F. de Juan, D. I. Pikulin, and M. Franz, 
Phys. Rev. Lett. 121, 036403 (2018).

[35] O. Can, E. M. Nica, and M. Franz, Phys. Rev. B 99, 045419 (2019).

[36] I. Danshita, M. Hanada, andM. Tezuka, Prog. Theor. Exp. Phys. 2017, $083 \mathrm{I} 01$ (2017).

[37] W. Fu and S. Sachdev, Phys. Rev. B 94, 035135 (2016).

[38] The real-SYK model we denoted here is slightly different with that in I. Danshita, M. Hanada, and M. Tezuka's work [36], where the variances of diagonal couplings are twice as the off-diagonal ones. In the numerical calculation, we do not find any significant difference between two setups.

[39] V. Oganesyan and D. A. Huse, Phys. Rev. B 75, 155111 (2007).

[40] Y. Y. Atas, E. Bogomolny, O. Giraud, and G. Roux, Phys. Rev. Lett. 110, 084101 (2013). 